PRINT ISSN 1119-8362

Electronic ISSN 1119-8362
Full-text Available Online at

https://www.ajol.info/index.php/jasem

http://ww.bioline.org.br/ja
J. Appl. Sci. Environ. Manage.

Vol. 24 (6) 975-978 June 2020

\title{
Classical Evaluation of Zero, Incomplete and Complete Treatment Effects on SEIRS Model Dynamics
}

\author{
*SANGOTOLA, AO; OYEWOLE, O \\ Department of physical sciences, Bells university of Technology, Ota, Ogun State. Nigeria. \\ *Corresponding Author Email: adekunle4000@hotmail.co.uk
}

\begin{abstract}
In this research work, we extend the classical SEIR model to accommodate the effect of zero, incomplete and complete treatment on the dynamics of the model. The basic reproduction number $\left(R_{0}\right)$ of the model dynamics is obtained by using the next generation matrix approach. The disease free equilibrium point of the model is found to be locally asymptotically stable if $R_{0}<1$. A suitable Lyapunov function is constructed to determine the global stability of the disease free equilibrium point. Numerical simulation is carried out to determine the effect on the compliance to the treatment prescription.
\end{abstract}

\section{DOI: https://dx.doi.org/10.4314/jasem.v24i6.5}

Copyright: Copyright (C) 2020 Sangotola and Oyewole. This is an open access article distributed under the Creative Commons Attribution License (CCL), which permits unrestricted use, distribution, and reproduction in any medium, provided the original work is properly cited.

Dates: Received: 13 April 2020; Revised: 17 May 2020; Accepted: 09 June 2020

Keywords: Equilibrium, Lyapunov function, Global stability, Simulation

The dynamics of infectious disease is best understood by their compartment structure (Brauer et al., 2010). The basic epidemiological compartment model divides the host population into susceptible, infectious and recovered population from which it can be extended to accommodate other compartments depending on the dynamics of the disease in question. Other components that can be added includes exposed, vaccinated, quarantined etc. Susceptible state is the state in which the host is not yet infected. Exposed state follows the susceptible state. The host is infected but cannot transmit the disease yet. Infectious state is the state where the host can transmit the disease while recovered state is the state where the host is no longer susceptible to the infection (Sangotola et al., 2019). The efficacy of treatment is one of the factors that determines if a disease will persist in a particular population (Okosun et al., 2011 and Zhang et al., 2014). Moreover, even with the availability of treatment, the attitude of the infectious population to the usage of the available treatment has an effect on the progression of the disease dynamics. Cai et al., (2013) rigorously analyzed a deterministic model with variable human population for malaria dynamics which allow transmission by recovered humans. The model analysis revealed the presence of backward bifurcation due to the presence of multiple endemic equilibrium. However, the backward bifurcation can be removed by replacing the standard incidence function with mass action incidence.
Ronoh et al., (2016) formulated a mathematical model for tuberculosis with drug resistance in the first line of treatment. They extended the standard SEIRS model of tuberculosis to include drug resistance. The basic epidemiological quantities were derived. Numerical simulation was carried out to determine the extent of drug resistance. Fred Brauer (2017) also outlined some of the important aspects of the development of mathematical epidemiology for present and future analysis.

Hence in this research work, we extend the classical SEIRS model to accommodate the effect of zero, incomplete and complete treatment on the dynamics on SEIR model. The basic epidemiological properties of the model is also determined.

\section{MATERIALS AND METHOD}

The model is divided into four compartments namely susceptible population denoted by $S(t)$, exposed population denoted by $E(t)$, infectious population denoted by $I(t)$ and recovered population denoted by $R(t)$. Susceptible population is increased as a result of migration or birth at rate $\Lambda$ and loss of immunity from recovered class at rate $\omega$. The exposed population is increased as a result of interaction between susceptible and infectious population at rate $\beta$ and by incomplete treatment of infectious population at rate $s$. There is an increase in the infectious population as a result of progression from exposed class at rate $\sigma$ while the recovered class is increased as a result of complete 
treatment at rate $r$. Every class is decreased by natural death at rate $\mu$ while the infectious class is further decreased as a result of zero treatment or complications from the disease at rate $d$. The parameter $s=(1-p) \theta$ and $r=p \theta$ where $\theta$ is the treatment function and $p$ is the probability that the treatment is effective. The value of the parameters used in the model formulation are approximated from most communicable diseases and it is given under the model simulation. The resulting system from the following assumptions is given as

$$
\begin{aligned}
& \frac{d S}{d t}=\Lambda-\beta S I-\mu S+\omega R \\
& \frac{d E}{d t}=\beta S I-(\mu+\sigma) E+s I \\
& \frac{d I}{d t}=\sigma \mathrm{E}-(\mu+d+r+s) I \\
& \frac{d R}{d t}=r I-(\omega+\mu) R
\end{aligned}
$$

\section{RESULTS AND DISCUSSIONS}

Existence and positivity of solution: The region where the model is mathematically and epidemiologically and mathematically well posed is established.

Theorem 1: (Existence and positivity of solution). The feasible region $\mathcal{R}$ defined by $\left\{S(t), E(t), I(t), R(t) \in R_{+}^{4}: N(0) \leq N(t) \leq \frac{\Lambda}{\mu}\right\}$ with initial conditions $S(0) \geq 0, E(0) \geq 0, I(0) \geq$ $0, R(0) \geq 0$ is positive invariant for system $(1)-(4)$.

Proof: The total population size is given by $N(t)=$ $S(t)+E(t)+I(t)+R(t)$

$$
\begin{gathered}
\frac{d N}{d t}=\Lambda-\mu N-d I \\
\frac{d N_{h}}{d t} \leq \Lambda-\mu N
\end{gathered}
$$

Solving above, we have

$$
\begin{aligned}
& 0 \leq N(t) \leq\left(N(0) e^{-\mu t}+\frac{\Lambda}{\mu}\right. \\
& t \rightarrow \infty, 0 \leq N(t) \leq \frac{\Lambda}{\mu} \text {. If } N(0) \leq \\
& N(0) \leq N(t) \leq \frac{\Lambda}{\mu}
\end{aligned}
$$

Thus, $\mathcal{R}$ is a positivity invariant set under the model described by (1) - (4).
Disease-free equilibrium point: The disease free equilibrium point is the point where there is no disease in the population. Hence it lies at the point

$$
\pi_{o}=\left(\frac{\Lambda}{\mu}, 0,0,0\right)
$$

Basic reproduction number: The basic reproductive number is the mean number of secondary infections caused by an infectious individual in an entirely susceptible population. The next generation matrix approach by Driessche and Watmough (2002) is applied. From the infectious stages $E$ and $I$, we can create a vector $\mathcal{F}$ that represents new infections and $\mathcal{V}$ that represents outflows from the model equations (Padmanabhan et al., 2017). Hence,

$\mathcal{F}=\left(\begin{array}{c}\beta S I \\ 0\end{array}\right)$ and $\mathcal{V}=\left(\begin{array}{c}(\mu+\sigma) E-s I \\ (\mu+d+r+s) I-\sigma \mathrm{E}\end{array}\right)$

Next, we compute the Jacobian $\mathrm{F}$ from $\mathcal{F}$ and $\mathrm{V}$ from $\mathcal{V}$ at the disease free equilibrium point. They are given by

$\mathrm{F}=\left(\begin{array}{cc}0 & \frac{\beta \Lambda}{\mu} \\ 0 & 0\end{array}\right)$ and $\mathrm{V}=\left(\begin{array}{cc}(\mu+\sigma) & -s \\ -\sigma & (\mu+d+r+s)\end{array}\right)$

The basic reproduction $R_{0}$ is given by $\rho\left(F V^{-1}\right)$ where $\rho$ is the spectral radius. The basic reproduction number is thus given by

$$
R_{0}=\frac{\beta \Lambda \sigma}{\mu[(\mu+\sigma)(\mu+d+r+s)-\sigma s]}
$$

Theorem 2: (Local stability of disease-free equilibrium). The disease-free equilibrium of the system (1) - (4) is locally asymptotically stable if $R_{0}<1$.

Proof: The Jacobian matrix evaluated at the diseasefree is given by

$$
\begin{aligned}
& J\left(\pi_{0}\right) \\
& =\left(\begin{array}{cccc}
-\mu & 0 & -\frac{\beta \Lambda}{\mu} & \omega \\
0 & -(\mu+\sigma) & \frac{\beta \Lambda}{\mu}+s & 0 \\
0 & \sigma & -(\mu+d+r+s) & 0 \\
0 & 0 & r & -(\omega+\mu)
\end{array}\right)
\end{aligned}
$$

There are four roots corresponding to the Jacobian above. Two of the roots are $-\mu$, and $-(\omega+\mu)$ while the other roots can be obtained from the characteristic equation of the sub matrix given below. 


$$
J_{1}\left(\pi_{0}\right)=\left(\begin{array}{cc}
-(\mu+\sigma) & \frac{\beta \Lambda}{\mu}+s \\
\sigma & -(\mu+d+r+s)
\end{array}\right)
$$

The remaining eigenvalues are negative if trace of $J_{1}\left(\pi_{0}\right)$ is negative and determinant of $J_{1}\left(\pi_{0}\right)$ is positive. Trace of $J_{1}\left(\pi_{0}\right)$ is negative. Hence, the determinant is $(\mu+\sigma)(\mu+d+r+s)-\left(\frac{\beta \Lambda}{\mu}+s\right) \sigma$ which is equivalent to the following equation:

$$
[(\mu+\sigma)(\mu+d+r+s)-\sigma s]\left(1-R_{0}\right)
$$

The determinant is positive provided that $R_{0}<1$.

Hence the disease-free equilibrium of the system (1) - (4) is locally asymptotically stable if $R_{0}<1$.

Theorem 3: (Global stability of disease-free equilibrium). The disease-free equilibrium of the system (1) - (4) is globally asymptotically stable if $R_{0}<1$.

Proof: Consider the Lyapunov function:

$V=\sigma E+(\mu+\sigma) I$

$\dot{V}=\sigma \dot{E}+\mu \dot{I}$

Substituting and simplifying the expressions for $\dot{E}$ and $\dot{I}$ gives

$\dot{V}$

$=[(\mu+\sigma)(\mu+d+r+s)$

$-\sigma s] I\left[\frac{\sigma \beta S}{[(\mu+\sigma)(\mu+d+r+s)-\sigma s]}-1\right]$

Since $S=\frac{\Lambda}{\mu} \leq N$, it follows that

$\dot{V} \leq[(\mu+\sigma)(\mu+d+r+s)-\sigma s] I\left[R_{0}-1\right]$

Thus $\dot{V} \leq 0$ with equality when $I=0$. Hence by LaSlle's extension to Lyapunov principle, the limit set for each solution is contained in the largest invariant set for which $I=0$.

Numerical Simulation: Numerical simulation is carried out to determine the effect of the treatment on the exposed and infectious class. The following estimated values which are approximated from most communicable diseases are used for model simulation: $\beta=0.12, \Lambda=0.002, \mu=0.00005, p=0.5, \sigma=$ $0.143, \theta=0.1, d=0.0025, E(0)=20$ and $I(0)=$ 20 . We can project the dynamics of treatment by varying $p$ on the interval $(0,0.5)$ and $(0.5,1)$. It is observed that irrespective of the value of $p$, there will be a rapid increase in both classes but the exposed class increased much more. The exposed class reaches its peak faster and its curve flattens faster. It is better to keep $p$ in the range $(0.5,1)$ to avoid an epidemic.

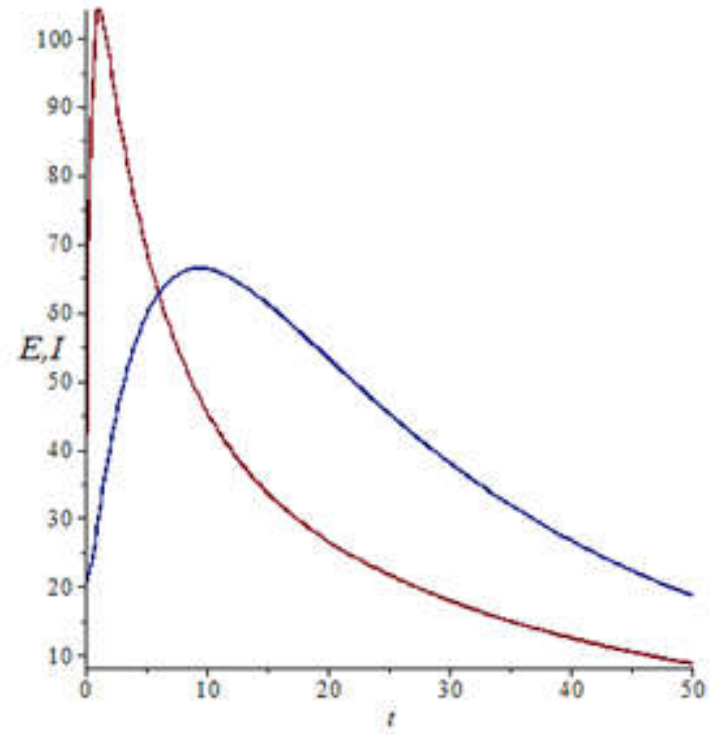

Fig 1: Numerical simulation between the exposed and infectious class

Conclusion: In this paper, we presented a SEIRS mathematical model with varying treatment dynamics. The basic reproduction number of the model is determined. The local and global stability of the disease free equilibrium points are determined. The implications of treatment variations is also examined.

\section{REFERENCES}

Brauer, F (2017). Mathematical epidemiology: Past, present, and future. Infectious Disease Modelling 2: 113-127.

Brauer, F; Chavez, C (2010). Mathematical Models in Population Biology and Epidemiology, Springer New York.

Brauer, F; Chavez, C (2013). Mathematical Models for Communicable Diseases, Society for Industrial and Applied Mathematics Philadelphia.

Cai, L; Lashari AA; Jung, IH; Okosun, KO; Seo, YI (2013). Mathemtical Analysis of a Malaria Model with partial immunity to reinfection. Hindawi Publishing Corporation Abstract and Applied Analysis Article ID 405258: 1-17.

Driessche, P; Watmough, J (2002). Reproduction numbers and subthreshold endemic equilibria for 
compartmental models of disease transmission. Math. Biosci. 180 (1): 29-48.

Ma, Z; Zhou, Y; Wu J (2009). Modelling and dynamics of infected disease, World Scientics Publishing Co Pte Ltd 5 Toh TuchLink, Singapore.

Okosun, KO; Makinde, DO (2011). Modelling the impact of drug resistance in malaria transmission and its optimal control analysis. International $J$. Phys. Sci. 6 (28): 6479-6487.

Padmanabhan, P; Seshaiyer, P (2017). Computational and Mathematical Methods to Estimate the Basic Reproduction Number and Final Size for SingleStage and Multistage Progression Disease Models for Zika with Preventative Measures. Compute. Math. Meth. Med. 25: 1-17.
Ronoh, M; Jaroudi, R; Fotso, P; Kamdoum, V; Matendecchere, N; Wairimu, J; Auma, R; Lugoye, J (2016). A Mathematical Model of Tuberculosis with Drug Resistance Effects. Applied Mathematics. 7 1303-1316.

Sangotola, AO; Onifade, AA (2019). A Generalized SEIR Mathematical Model with Infectivity in exposed period. Journal of the Nigerian Mathematical Society. 38 (1): 45-54.

Zhang, J; Jia, J; Song, X (2014). Analysis of an SEIR Epidemic Model and Saturated Treatment Function. Hindawi Publishing Corporation The Scientific World Journal Article ID 910421: 1-11. 